Perspective taking reduces the fundamental attribution error

Abstract

The fundamental attribution error (FAE) refers to the predisposition for people to

3 attribute the behavior of others to dispositional characteristics, rather than situational

4 causes external to the individual. The current study aimed to investigate whether pre-

5 experimental perspective taking (PT) training could reduce the FAE. Participants were

6 randomly assigned to either receive PT training, or to receive no training, before

7 completing a typical attitude attribution task. This task required participants to watch a

8 video clip of an actor reading an essay for or against capital punishment and then to infer

9 the attitude of the actor. Results indicated that participants in the perspective taking

10 condition experienced a significant reduction in the FAE compared to participants in the

11 control condition.

12

13

14

15

16

17

18

19

20

21

22

23

24

25 
Perspective taking reduces the fundamental attribution error

\section{PERSPECTIVE TAKING REDUCES THE FUNDAMENTAL ATTRIBUTION ERROR}

When assessing and forming judgements of an individual's behavior we tend to overlook contextual information and attribute behavior to internal dispositions (Gawronski, 2004; Masuda \& Kitayama, 2004; Gilbert \& Malone, 1995; Ross, Green, \& House, 1977).

This bias, which has been well documented in the social psychology literature and is referred to as the fundamental attribution error (FAE), can have significant negative consequences (Alicke, 2000; Gilbert \& Malone, 1995).

Perspective taking, which can be defined as adopting another person's viewpoint (Parker \& Axtell, 2001), may be a way to reduce the FAE. For example, a person with well-developed PT skills should be able to view a situation from the perspective of another individual and thus anticipate their beliefs, desires, emotions and intentions (Epley, Morewedge, \& Keysar, 2004). Perspective taking has been empirically implicated in various ways; it has been recommended as a simple strategy for reducing social bias and for strengthening the creation and maintenance of social bonds (Galinsky, Ku, \& Wang, 2005), it has been used to reduce stereotyping (Yee \& Bailenson, 2006) and it has been shown to improve negotiation skills (Galinsky \& Mussweiler, 2001). Of particular interest to the current study, Storms (1973) investigated an experimental manipulation of visual orientation in the attribution process. In other words, the researcher altered the viewpoint of an observer prior to an attitude attribution task and found dispositional inferences made by subjects were reduced.

A recent functional analytic theory is gaining increasing empirical support for its account of perspective taking as a form of learnt or operant behavior (e.g., McHugh, Barnes-Holmes, \& Barnes-Holmes, 2004; McHugh \& Stewart, 2012). This account is referred to as Relational Frame Theory (RFT: Hayes, Barnes-Holmes, \& Roche, 2001). According to this approach the key to human language and cognition is the ability to put 
Perspective taking reduces the fundamental attribution error

51 things into relations with each other not based on their physical properties but based on cues as to which relation to apply. This is called relational framing. Consider the relation between a word and an object. This is perhaps the most fundamental and important aspect of language. An example would be the relation between the word 'ball' and an actual 'ball'. Humans can treat these two things as being the same as each other, despite the fact that they are not physically the same as each other. For instance, if I ask you to give me the ball, you will hand me the actual ball. In other words, humans can put these things into an abstract relation of sameness with each other. Sameness is just one example of relational framing. There are other examples that we go on to learn; comparison, opposition, difference, temporal, spatial and hierarchical.

RFT suggests that we learn to relate (relationally frame) things in our environment and that this relational activity can change the psychological functions of those things. This change in psychological functions is referred to as 'transformation of function' (TOF) and this effect can be useful in many contexts (see Dymond \& Roche, 2013). However, TOF can also be problematic in some contexts. For example, I may frame myself as a socially awkward individual and based on that framing I may derive further relations such that I should avoid company. In the latter example, the functions of other people are transformed for me so that I tend to avoid them, even though interaction with them might be psychologically beneficial.

\section{Children learn to relate their own behavior as different from that of others by} learning three key 'deictic' or 'perspective' relations which are "I versus YOU”, "HERE versus THERE" and "NOW versus THEN". They learn to respond appropriately to questions such as 'What are YOU doing HERE?', 'What am I doing NOW?', 'What was I doing THEN?' etc. As children gradually learn to respond appropriately to these questions, and as they learn that whenever they are asked about their own behavior they always 
Perspective taking reduces the fundamental attribution error

answer from the point of view of 'I', 'HERE' and 'NOW', they will learn this perspective is consistent and different from that of other people. For example, if you ask an individual about their own behaviour, s/he will always answer from the position of 'I', 'HERE' and 'NOW' in response to your question asked by YOU, THERE (where you are) and THEN (when you asked - a few seconds ago). I is always from this perspective here, not from someone else's perspective there. A sense of perspective is therefore abstracted through learning to talk about one's own perspective in relation to other perspectives. Previous research in this area has demonstrated that perspective taking can be trained when deficient (Weil, Hayes, \& Capurro, 2011), that rehearsing perspective taking can enhance the repertoire on a subsequent task (Vilardaga, Estévez, Levin, \& Hayes, 2012) and that an under rehearsal of this repertoire can result in perspective taking deficits (Janssen, et al., 2014).

The current study aims to develop the RFT literature on perspective taking by asking participants to engage in PT training (McHugh et al., 2004) prior to completing the most widely employed test of the FAE; the attitude attribution paradigm (Bauman \& Skitka, 2010; Jones \& Harris, 1967; Wright \& Wells, 1988). We predict that the perspective taking groups will experience a reduction in the FAE. (1)

Method

\section{Participants and Design}

A total of 80 participants from the general public (i.e., 50 females and 30 males) took part in the experiment. All participants were over the age of 18 years old (mean 25.23 years, $\mathrm{SD}=10.71$ ) and were assigned to one of four groups via the excel random number generator. Each group therefore had 20 participants. Group 1 (14 females; mean 24.65 years, $\mathrm{SD}=11.01$ ) received $\mathrm{PT}$ training and watched a video 'for' capital punishment 
Perspective taking reduces the fundamental attribution error

101 Group 2 (12 females; mean 26.95 years, $\mathrm{SD}=12.98)$ received PT training and watched a

102 video 'against' capital punishment. Group 3 (11 females; mean 22.25 years, SD=0.77)

103 received no training and the 'for' capital punishment video. Group 4 (13 females; mean

10427.05 years, $\mathrm{SD}=12.36$ ) received no training and the 'against' capital punishment video.

105 The study employed a 2 (training: PT training vs. no training) x 2 (position: for vs. against)

106 between subjects design, with FAE score as the dependent variable.

Stimulus

Perspective Taking (PT) Training. Participants in the PT groups received a training

110 exercise (McHugh, et al., 2004, protocol) consisting of 30 questions with 2 answers to

111 choose from. The protocol involved trials that required the participant to respond to the

112 three perspective-taking frames of I-YOU, HERE-THERE and NOW-THEN across three

113 levels of relational complexity (i.e., a simple relational response; a reversed relational

114 response; and a double reversed relational response). For example, a simple NOW THEN

115 trial is as follows: 'Yesterday I was watching television, today I am reading a book. What

116 am I doing now?' A reversed I YOU trial is as follows: 'I have a red brick and you have a

117 green brick. If I was you and you were me, what would you have?' Finally, a double

118 reversed HERE THERE, NOW THEN trial is as follows: 'Yesterday you were sitting here

119 on the blue chair and today you are sitting there on the black chair. If now was then and

120 then was now and here was there and there was here. Where would you be sitting today?'

121 Participants were free to answer these questions in as much time as they needed. In line

122 with McHugh et al. (2004) and Villatte, Monestes, McHugh, Freixa i Baqué and Loas

123 (2010) accuracy rates of 50\% in the two-response protocol can be interpreted as chance

124 level responding therefore only participants whose scores were over $67 \%$, and thus

125 demonstrated adherence to the perspective taking protocol were included in the analysis. 
Perspective taking reduces the fundamental attribution error

126 Only 3/40 participants did not meet this criterion, one 'for' and two 'against' (see

127 Appendix 1 for participants overall accuracy on the perspective taking protocol).

128 Attitude Attribution Task. Participants were asked to watch either a 'for' or 'against'

129 capital punishment video clip. The video clips were created with the help of a female

130 confederate who read the essays (taken from Masuda \& Kitayama, 2004) without emotion.

131 The 'against' capital punishment video was 1 minute 37 seconds, whilst the 'for' capital

132 punishment video was 1 minute 29 seconds.

133 Following the video participants answered an FAE questionnaire, which consisted

134 of three questions (taken from Masuda \& Kitayama, 2004). Question 1 was 'please infer

135 the attitude of the individual in the video towards capital punishment' where 1 was equal to

136 'extremely against' and 15 was equal to 'extremely in favour'. If participants recorded

137 scores closer to the extremities of the scale on this measure then they committed the FAE.

138 Question 2 asked participants to estimate the attitude of an average 20 year old on the topic

139 of capital punishment and Question 3 asked participants to indicate their own views on

140 capital punishment. These questions were also assessed on a 15-point scale. As with

141 Masuda and Kitayama (2004) estimates of both average attitude and the participants own

142 views were negative towards capital punishment, but they bore no relationship to the

143 results recorded on the FAE measure. Hence they will not be discussed further.

\section{Procedure}

146 After being randomly assigned to condition, participants in the perspective taking 147 groups were told that the experiment would involve two unrelated parts; firstly they would 148 have to complete a 'cognitive measure' and secondly they would have to complete an 149 everyday decision making task that required them to watch a video about capital 150 punishment and rate their opinion on the topic. Those in the control group received no 
Perspective taking reduces the fundamental attribution error

151 training so were only given the second part. Before beginning the attitude attribution task

152 all participants were made explicitly aware that the speaker would be reading an essay that

153 they were assigned to write: "The experiment concerns attitude inference. The person

154 speaking in the video will be reading an essay for or against capital punishment that they

155 were assigned to write in an English class". See Figure 1 for a graphical representation of

156 the procedure.

157

158

Insert Figure 1

159

160

Results

161

Figure 1 suggests that the control group in both the 'for' $(\mathrm{M}=11.85, \mathrm{SD}=2.36)$

162 and 'against' $(\mathrm{M}=3.5, \mathrm{SD}=2.92)$ capital punishment conditions committed greater FAE

163 than the participants who received PT training in the 'for' $(\mathrm{M}=10.05, \mathrm{SD}=2.55)$ and

164 'against' $(\mathrm{M}=5.22, \mathrm{SD}=3.57)$ capital punishment conditions.

165

Insert Figure 2

167

168

169

170

171 were closer to 8 in the perspective taking groups).

Discussion 
Perspective taking reduces the fundamental attribution error

The current experiment indicated that a pre-experimental perspective taking exercise reduced the fundamental attribution error. The current findings have implications both at a practical and theoretical level. At a practical level, the results suggest that brief perspective taking interventions could have use in improving everyday social interactions in which the FAE is committed. Indeed such exercises would be easily disseminable and could be accomplished in many different contexts (from schools to workplaces).

At a theoretical level, the current study demonstrated the effectiveness of exposing participants to an RFT based perspective-taking protocol. According to RFT, the core of language is being able to put things into abstract relations that do not depend on the characteristics of the things being related but instead depend on cues that 'signal' which relational frame is appropriate. There is increasing evidence for these frames (e.g., Steele \& Hayes, 1991). As we learn to respond to perspective relations (i.e., I YOU, HERE THERE and NOW THEN) we gradually learn to abstract a sense of perspective so that whenever we are asked about our own behavior we learn to answer from the point of view of 'I', 'HERE' and 'NOW' and we learn that this perspective is consistent and different from that of other people. The key advantage of the RFT approach to understanding and developing perspective taking is that RFT is a behavioral approach to explaining behaviour, which means that it is a naturalistic, empirical and pragmatic approach.

It is naturalistic because it is not based on things that cannot be directly seen or manipulated, such as the id or the ego of psychodynamics or the visual-spatial sketchpad of cognitive psychology, for example. Instead, its theoretical explanations always include processes in the environment that affect behavior and that can be directly seen and manipulated by the scientist. For example, relational responding is a measurable activity that is affected by socio-verbal interaction and indeed, as suggested above, can be trained by systematically changing the environment (e.g., by focusing on particular types of 
Perspective taking reduces the fundamental attribution error

199

200

201

202

203

204

relations). It is empirical as it is based on a scientific theory developed following the observations of scientists over decades of behavior analytic research, and in that time there has been substantial empirical and theoretical progress (see Dymond \& Roche, 2013 for a recent book length review of this progress). Finally, it is pragmatic as it aims to actually change behavior, not simply describe it. In fact this intentional focus on changing behavior is a fundamental, 'built-in' feature of this account and therefore it will continue to lead to immediate and promising applications.

There are a number of limitations to the current study that would need to be addressed in future research. Firstly, no measure of state perspective taking ability was taken following the intervention to ensure that that those in the perspective taking groups were, in fact, better able to take perspective of others than those in the control group. However, given that there is no standardized state scale of perspective taking ability that could be used to assess this, other investigations have yet to include such a measure in research of this kind (Vilardaga et al., 2012). Secondly, although every effort was made to convince the perspective taking groups that the training was unrelated to the subsequent FAE task, it is possible that exposure to such an intervention may have primed the participants to be more careful during the FAE task, not as a function of increased perspective taking abilities, but because they became more suspicious following the task. In order to overcome this issue it may be worthwhile to repeat the investigation with a control group who do mock perspective taking training where the three relational abilities are not targeted. However, it is important to note that past research has employed such control groups and found no difference between a mock control group and a no training control group (Weger, Hooper, Meier, \& Hopthrow, 2012).

Future research could include a pre-experimental measure of perspective taking and then use moderation analysis to investigate the effect of PT training on the FAE. It may 
Perspective taking reduces the fundamental attribution error

224 also be interesting to determine the effects of an extended perspective taking training exercise. For example, it is likely that longer perspective taking training may result in a greater ability to appreciate the contextual variables in a given situation. Overall, this is the first study to attempt to use perspective taking training to attenuate the FAE. The results are particularly noteworthy given that the findings reported herein suggest that a brief exercise in taking the perspective of another may be useful in reducing the robust FAE phenomena.

\section{References}

Alicke, M. D. (2000). Culpable control and the psychology of blame. Psychological bulletin, 126(4), 556.

Bauman, C. W., \& Skitka, L. J. (2010). Making attributions for behaviors: the prevalence of correspondence bias in the general population. Basic and Applied Social Psychology, 32(3), 269-277.

Dymond, S., \& Roche, B. (2013). Advances in Relational Frame Theory: Research and Application. Oakland, CA: New Harbinger Publications

Epley, N., Morewedge, C. K., \& Keysar, B. (2004). Perspective taking in children and adults: Equivalent egocentrism but differential correction. Journal of Experimental Social Psychology, 40(6), 760-768.

Galinsky, A. D., \& Mussweiler, T. (2001). First offers as anchors: the role of perspectivetaking and negotiator focus. Journal of personality and social psychology, 81(4), 657.

245 Galinsky, A. D., Ku, G., \& Wang, C. S. (2005). Perspective-taking and self-other overlap: Fostering social bonds and facilitating social coordination. Group Processes \& Intergroup Relations, 8(2), 109-124. 
Perspective taking reduces the fundamental attribution error

248 Gawronski, B. (2004). Theory-based bias correction in dispositional inference: The fundamental attribution error is dead, long live the correspondence bias. European review of social psychology, 15(1), 183-217.

Gilbert, D. T., \& Malone, P. S. (1995). The correspondence bias. Psychological bulletin, $117(1), 21$.

Hayes, S. C., Barnes-Holmes, D., \& Roche, B. (2001). Relational Frame Theory: A postSkinnerian account of human language and cognition. New York: Plenum.

Janssen, G., De Mey, H., Hendriks, A., Koppers, A., Kaarsemaker, M., Witteman, C., \& Egger, J. (2014). Assessing Deictic Relational Responding in Individuals With Social Anxiety Disorder: Evidence of Perspective-Taking Difficulties. The

Jones, E. E., \& Harris, V. A. (1967). The attribution of attitudes. Journal of experimental Psychological Record, 64(1), 21-29. social psychology, 3(1), 1-24.

Masuda, T., \& Kitayama, S. (2004). Perceiver-induced constraint and attitude attribution in Japan and the US: A case for the cultural dependence of the correspondence bias.

264 McHugh, L., \& Stewart, I. (2012). The Self and Perspective Taking: Research and Applications.

McHugh, L., Barnes-Holmes, Y., \& Barnes-Holmes, D. (2004). Perspective-taking as relational responding: A developmental profile. The Psychological Record, 54(1),

269 Parker, S. K., \& Axtell, C. M. (2001). Seeing another viewpoint: Antecedents and outcomes of employee perspective taking. Academy of Management Journal, 44(6), 1085-1100. 
Perspective taking reduces the fundamental attribution error

272 Steele, D., \& Hayes, S. C. (1991). Stimulus equivalence and arbitrarily applicable relational responding. Journal of the Experimental Analysis of Behavior, 56(3), 519-555.

274 Storms, M. D. (1973). Videotape and the attribution process: reversing actors' and observers' points of view. Journal of personality and social psychology, 27(2), 165.

Vilardaga, R., Estévez, A., Levin, M. E., \& Hayes, S. C. (2012). Deictic Relational Responding, Empathy, and Experiential Avoidance as Predictors of Social

Villatte, M., Monestès, J. L., McHugh, L., Freixa i Baqué, E., \& Loas, G. (2010). Assessing perspective taking in schizophrenia using Relational Frame Theory. The Psychological Record, 60, 413-424.

Weger, U. W., Hooper, N., Meier, B. P., \& Hopthrow, T. (2012). Mindful maths: Reducing the impact of stereotype threat through a mindfulness exercise. Consciousness and cognition, 21(1), 471-475.

Weil, T. M., Hayes, S. C., \& Capurro, P. (2011). Establishing a deictic relational repertoire in young children. The Psychological Record, 61(3), 5.

288 Wright, E. F., \& Wells, G. L. (1988). Is the attitude-attribution paradigm suitable for investigating the dispositional bias?. Personality and Social Psychology Bulletin,

291 Yee, N., \& Bailenson, J. N. (2006). Walk a mile in digital shoes: The impact of embodied perspective-taking on the reduction of negative stereotyping in immersive virtual environments. Proceedings of PRESENCE, 24-26.

\section{Appendix 1}

295 Accuracy scores across the two Perspective Taking Groups.

296 For $\quad$ Against 
Perspective taking reduces the fundamental attribution error

\begin{tabular}{|c|c|c|}
\hline 297 & 27 & 26 \\
\hline 298 & 27 & 26 \\
\hline 299 & 21 & 30 \\
\hline 300 & 26 & 26 \\
\hline 301 & 28 & 28 \\
\hline 302 & 23 & 29 \\
\hline 303 & 29 & 30 \\
\hline 304 & 29 & 30 \\
\hline 305 & 30 & 29 \\
\hline 306 & 30 & 29 \\
\hline 307 & 29 & 30 \\
\hline 308 & 28 & 29 \\
\hline 309 & 29 & 29 \\
\hline 310 & 28 & 29 \\
\hline 311 & 30 & 30 \\
\hline 312 & 20 & 28 \\
\hline 313 & 23 & 30 \\
\hline 314 & 29 & 30 \\
\hline 315 & 27 & \\
\hline 316 & & \\
\hline
\end{tabular}

